OPEN ACCESS

Edited by:

Steven Hirschfeld,

Uniformed Services University of the

Health Sciences, United States

Reviewed by:

Connie M. Weaver,

Purdue University, United States

Vallo Tillmann,

University of Tartu, Estonia

*Correspondence:

Päivi M. Paldánius

paivi.m.paldanius@helsinki.fi

Specialty section:

This article was submitted to

Children and Health,

a section of the journal

Frontiers in Pediatrics

Received: 25 September 2020

Accepted: 16 July 2021

Published: 24 August 2021

Citation:

Paldánius PM, Ivaska KK, Mäkitie O and Viljakainen $H$ (2021) Serum and Urinary Osteocalcin in Healthy 7- to

19-Year-Old Finnish Children and Adolescents.

Front. Pediatr. 9:610227. doi: 10.3389/fped.2021.610227

\section{Serum and Urinary Osteocalcin in Healthy 7- to 19-Year-Old Finnish Children and Adolescents}

\author{
Päivi M. Paldánius ${ }^{1,2 *}$, Kaisa K. Ivaska ${ }^{3}$, Outi Mäkitie ${ }^{1,2,4,5}$ and Heli Viljakainen ${ }^{5,6}$ \\ ${ }^{1}$ Children's Hospital, University of Helsinki and Helsinki University Hospital, Helsinki, Finland, ${ }^{2}$ Research Program for Clinical \\ and Molecular Metabolism, University of Helsinki, Helsinki, Finland, ${ }^{3}$ University of Turku, Institute of Biomedicine, Turku, \\ Finland, ${ }^{4}$ Department of Molecular Medicine and Surgery, Center for Molecular Medicine, Karolinska Institutet, and Clinical \\ Genetics, Karolinska University Hospital, Stockholm, Sweden, ${ }^{5}$ Folkhälsan Research Center, Helsinki, Finland, ${ }^{6}$ Department \\ of Food and Nutrition, University of Helsinki, Helsinki, Finland
}

Children and adolescents have high bone turnover marker (BTM) levels due to high growth velocity and rapid bone turnover. Pediatric normative values for BTMs reflecting bone formation and resorption are vital for timely assessment of healthy bone turnover, investigating skeletal diseases, or monitoring treatment outcomes. Optimally, clinically feasible measurement protocols for BTMs would be validated and measurable in both urine and serum. We aimed to (a) establish sex- and age-specific reference intervals for urinary and serum total and carboxylated osteocalcin (OC) in 7- to 19-year-old healthy Finnish children and adolescents $(n=172)$, (b) validate these against standardized serum and urinary BTMs, and (c) assess the impact of anthropometry, pubertal status, and body composition on the OC values. All OC values in addition to other BTMs increased with puberty and correlated with pubertal growth, which occurred and declined earlier in girls than in boys. The mean serum total and carboxylated $\mathrm{OC}$ and urinary $\mathrm{OC}$ values and percentiles for sex-specific age categories and pubertal stages were established. Correlation between serum and urinary OC was weak, especially in younger boys, but improved with increasing age. The independent determinants for $\mathrm{OC}$ varied, the urinary OC being the most robust while age, height, weight, and plasma parathyroid hormone (PTH) influenced serum total and carboxylated OC values. Body composition parameters had no influence on any of the OC values. In children and adolescents, circulating and urinary $\mathrm{OC}$ reflect more accurately growth status than bone mineral density (BMD) or body composition. Thus, validity of OC, similar to other BTMs, as a single marker of bone turnover, remains limited. Yet, serum and urinary OC similarly to other BTMs provide a valuable supplementary tool when assessing longitudinal changes in bone health with repeat measurements, in combination with other clinically relevant parameters.

Keywords: pediatric normative values, bone turnover markers, serum osteocalcin, urinary mid-fragment osteocalcin, sex and age-specific ranges 


\section{INTRODUCTION}

Normal pediatric reference ranges for bone turnover markers (BTMs), reflecting bone formation and resorption, are a prerequisite for timely assessment of metabolic bone disorders and monitoring of response to therapy or disease progression (1). As compared with adults, children and adolescents have elevated circulating concentrations of BTMs, reflecting sequential periods of high growth velocity and rapid bone turnover $(2,3)$. The highest levels of BTMs have been described in infants and children during the first 4 years of life $(2,4,5)$; thereafter, marker levels decrease slightly and remain relatively stable until peaking again at puberty (6). Puberty is a crucial period for final bone mass accrual (7), with $25 \%$ increase in lumbar spine volumetric bone mineral density (BMD) during puberty (8). Nevertheless, there appears to be no relationship between the length of puberty and accrual of peak bone mass (9).

Net gain in bone mass in childhood results from bone modeling. Even if also bone resorption takes place during this time, the bone mass accrual is driven by the rapid bone formation, which by far exceeds the rate of resorption (10). The subsequent increase in BTMs should coincide with the pubertal growth spurt while increased variation in BTM levels also demonstrates correlation with growth velocity. This fluctuation surges during pubertal years due to considerable changes in absolute measured concentrations of both formation and resorption markers with age (10-12). Generally, almost $90 \%$ of the skeletal mass is being attained by 18 years (13), even if the process greatly varies between individuals, and is influenced by many physiological and pathological factors such as genetics, gender, ethnicity, prematurity, endocrine and mechanistic factors, (mal)nutrition, and pharmacological factors (14). Consequently, any longitudinal measurement to distinguish between normal and abnormal bone turnover in a growing child necessitates comparison against pediatric normative reference curves.

BTMs are specific bone-derived proteins or their fragments, which are present in serum and/or urine, often classified as markers reflecting either bone formation or bone resorption, and their concentrations reflect bone metabolic activity in total body at a given time point. The use of a composite set of formation and resorption markers instead of single markers is recommended in the longitudinal assessment of bone metabolism, as the sensitivity and/or specificity of any single marker is usually poor $(1,15)$.

Osteocalcin (OC) is a bone-derived protein, a widely used marker of bone formation in adults, which has also been shown to correlate with serum testosterone and bone maturation in boys (16). Previous pediatric studies have demonstrated that serum OC is a sensitive marker of skeletal growth in healthy children and in those with pathologically increased growth velocity (17). OC is metabolized in kidneys as measurable mid-molecule fragments. The urinary levels of OC correlate well with serum OC concentrations and other serum BTMs in adults (18). Urinary mid-molecular fragments of OC have been used alone in adults as an index of bone turnover but have not yet been established as a marker of bone turnover in children and adolescents. In one study, the urinary mid-fragments predicted catch up growth in prematurely born infants suggesting that it may be a valid marker even in children (19).

Increasing adiposity has been suggested as a risk factor for fractures in children $(20,21)$ while the role of BMI in extremity fractures is unclear (22). Studies in young adults, with or without impaired glucose tolerance or diabetes (23-25), indicate that OC may play a potential role in energy metabolism, even if the results are inconsistent and require further validation (26). In reverse, poor glycemic and metabolic control may affect bones and/or bone cell function $(27,28)$. Thus, additional adjustment of BTM reference range data for metabolic factors and adiposity (BMI and body composition), as well as exclusion of individuals with diabetes or impaired glucose tolerance, provides a foundation for correct interpretation of BTM results in healthy pediatric subjects (29).

Here, we aimed to establish sex- and age-specific reference intervals for serum and urinary OC in healthy Finnish children and adolescents (29). We assessed the impact of anthropometry, pubertal status, and fat percentage and body composition on creatinine corrected urinary and serum total and serum carboxylated OC levels. Additionally, we evaluated the concentrations of standardized, validated urinary and serum BTMs reflecting bone formation and resorption for each age cohort, separately for boys and girls.

\section{MATERIALS AND METHODS}

\section{Participants}

The original study comprised 195 children and adolescents who were included in a school-based cross-sectional study in the capital region of Helsinki $\left(60^{\circ} \mathrm{N}\right)$, in southern Finland. As previously described (30), the participants were recruited from randomly selected school classes in one primary and one secondary school for sufficient coverage of all the age groups (7-18 years) and aiming at higher than $60 \%$ participation rate. However, no strata for inclusion of all ages and sex were being introduced. Participation in this study, which was designed to assess the relationship between Vitamin D and aspects of bone health, was voluntary and invitation letters were given by the teachers to the pupils and their parents. All those willing to participate were included.

This current study included a total of 172 subjects from the original cohort, 106 girls and 66 boys, who presented with normal $\mathrm{BMD}$, i.e., whole body (WB) BMD $Z$-score between -2.0 and +2.0 , and had data for clinical characteristics, including puberty stage and at least sufficient serum samples for OC analysis. One outlier (an adolescent boy) was excluded due to an unreliable test result for an exceptionally high urinary OC value.

\section{Ethics Statement}

The Helsinki and Uusimaa Hospital District Independent Ethics Committee approved the study protocol and a written, ageadjusted informed consent was given by the participants and/or their caregivers, as appropriate. The study was carried out according to the principles of the Declaration of Helsinki. 


\section{Clinical Characteristics}

All 172 subjects were evaluated for anthropometry (weight, height, and BMI). As described elsewhere (30), the subjects completed a questionnaire on medical and fracture history, medications, overall health, age at menarche for girls, use of vitamin $\mathrm{D}$, and calcium supplements, and data regarding physical activity and dietary intakes were collected with a semiquantitative food frequency questionnaire. Height $(\mathrm{cm})$ and weight $(\mathrm{kg})$ were measured and compared with Finnish growth charts and height standard deviation (SD) score (height $Z$-score) was defined as deviation of height, in SD units, from the mean height for age and sex $(31,32)$. We included no separate analysis on ethnicity due to limited ethnic heterogeneity (>90\% were ethnic Caucasians/Finns).

Based on questionnaire data, serum gonadotropin and sex steroid concentrations (estradiol and testosterone), and information on menarche, pubertal development was scored either as pre-, mid-, or post-pubertal by an experienced pediatric endocrinologist (OM) (30). A united scale was introduced by converting the available Tanner stages (33) into pre-, mid-, or post-pubertal categories as follows: Tanner stages I and II were considered as pre-pubertal, stages III and IV were considered as mid-pubertal, and stage $\mathrm{V}$ was considered as post-pubertal.

\section{Biochemistry}

Blood samples and second void urine were collected between 8:00 a.m. and 10:00 a.m. after an overnight fast between November and March (winter months). Serum 25-hydroxyvitamin D (25-OHD) was assayed with highperformance liquid chromatography (HPLC, evaluated by Vitamin D External Quality Assessment Scheme, DEQAS), and plasma fasting parathyroid hormone (PTH) was assayed by an immunoluminometric method.

\section{Serum and Urinary OC}

Serum total OC and serum $\gamma$-carboxylated OC levels were determined as previously described by two-site immunoassay (34). This method for serum total OC is based on monoclonal antibodies detecting the $\mathrm{N}$-terminal mid-segment of the $\mathrm{OC}$ molecule. Assay for carboxylated OC detects the same fragments but prefers $\gamma$-carboxyglutamic acid (Gla) containing forms of OC, with $<10 \%$ cross-reactivity to fully uncarboxylated OC (35). Synthetic peptide of human OC amino acids 1-49 (Advanced Chemtech, Louisville, KY, USA) was used as a calibrator. The reported within-assay and between-assay variations (CV) for the assays are $<5$ and $<8 \%$ (35).

Urinary mid-fragment OC was determined with the previously described two-site immunoassay for the OC midfragment that uses synthetic peptide of human OC amino acids 1-43 (Advanced Chemtech, as above) as a calibrator (18). The detection limit for the urinary $\mathrm{OC}$ assay is $0.2 \mathrm{mg} / \mathrm{L}$ and the intra-assay and inter-assay CVs in this cohort were 1.5 and $3.4 \%$, respectively. All the urinary $\mathrm{OC}$ values were corrected for urinary creatinine (nM; as analyzed according to standard protocols of the Central Laboratory of Helsinki University Central Hospital). All OC samples were measured as duplicates and simultaneously at the end of the study.

\section{Other BTMs}

Serum intact N-terminal Propeptides of Type I Collagen (SP1NP), C-Terminal Telopeptide of Type I Collagen (S-ICTP), and alkaline phosphatase (S-ALP) were determined per validated standard protocols of the Central Laboratory of Helsinki University Central Hospital as described elsewhere $(30,36)$. Concentration of N-Terminal Telopeptide of Type I Collagen (U-NTx) was measured from second void urine samples with Osteomark ${ }^{\circledR}$ NTx Urine Enzyme-linked Immunosorbent Assay (Alere Scarborough, Inc., Scarborough, USA) with intraand inter-assay $\mathrm{CV} \%<8$. The given values (nM Bone Collagen Equivalents) were corrected with urinary creatinine concentration (nM).

\section{Analysis of Skeletal Characteristics and Body Composition}

$\mathrm{BMD}$, bone mineral content (BMC), and bone area (BA) were measured with dual-energy X-ray absorptiometry (DXA, Hologic Discovery A, pediatric software, version 12.4) from the lumbar spine (LS) (L1-L4), total hip, and whole body (WB). DXA measurements were performed within 3 months of the biochemical sampling. All BMD values were transformed into $Z$-scores using the equipment-specific age- and sex-adjusted reference data for US Caucasian children (37). Body composition was assessed simultaneously with DXA to obtain lean body mass and whole-body fat mass (WB fat percentage; WB fat\%). Calibration of the measurements was performed by using a spine phantom; inter-CV\% for the phantom BMC, area, and BMD were $0.35,0.21$, and $0.41 \%$, respectively. The reproducibility of the DXA measurement for bone, fat, and lean mass is 1.2, 1.9, and $0.7 \%$, respectively, in children between 10 and 18 years of age (38).

\section{Statistical Analysis}

Baseline demographics and all the BTMs, separately for both girls and boys, were reported as descriptive statistics with means and 95\% confidence intervals (CIs), standard error of the mean, and range (minimum, maximum). Additionally, Tukey's Hinges percentiles were calculated for serum and urinary OC parameters. OC data were non-normally distributed (ShapiroWilk test $<0.95)$ requiring logarithmic transformation; back-transformed data curves and values are presented here for practical use. Tertiles of Vitamin D concentrations were used to evaluate the relationship between the 25-OHD concentrations and PTH or BTMs in a univariate analysis (ANOVA), followed by Bonferroni (normally distributed data) or non-parametric Kruskal-Wallis (not normally distributed data). Multivariable regression analysis was used to identify independent determinants influencing BTM values in the overall pooled population. The covariates included anthropometric factors and their derivates such as age, sex, weight, height, BMI, height SD, weight percentage, puberty status, $\mathrm{PTH}$, serum 25 -OHD, whole body fat percentage, whole body weight $Z$-score, lumbar $Z$-score, and femoral $Z$-score. Simple regression analysis was first performed to screen potential predictors for OCs and a multivariate stepwise linear regression model was used to identify and determine significant predictors for BTMs. Using 


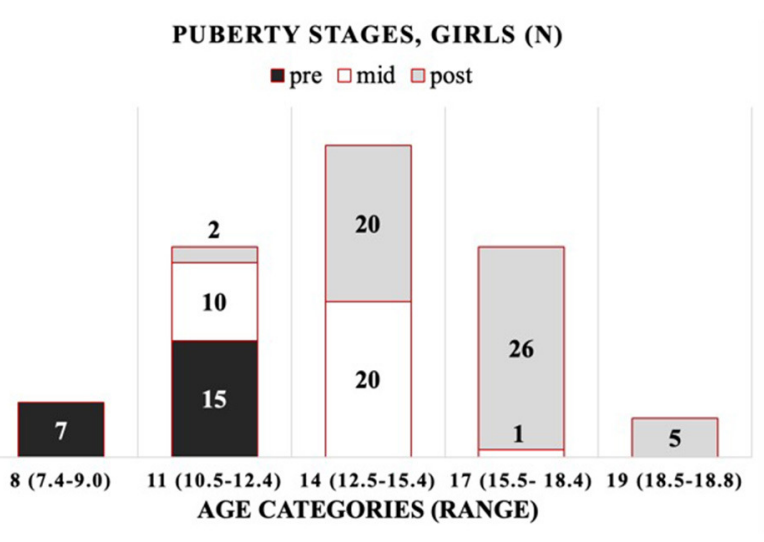

FIGURE 1 | Progression of puberty status per age category for girls ( $n$ per puberty category)

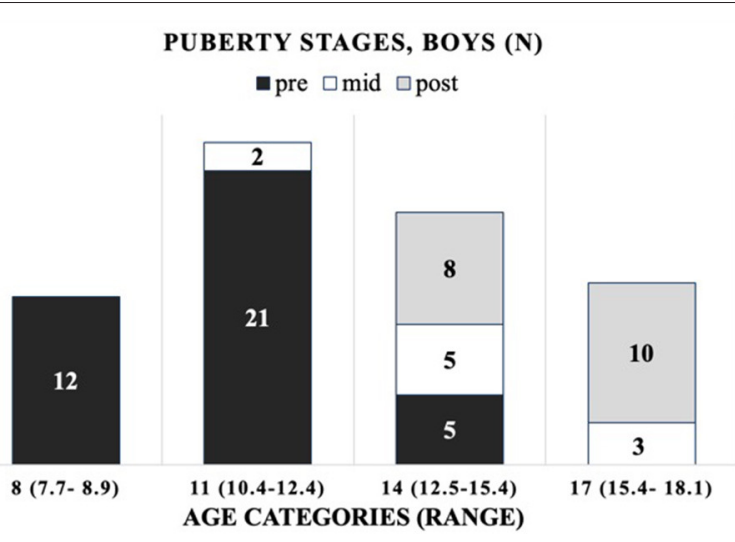

FIGURE 2 | Progression of puberty status per age category for boys ( $n$ per puberty category). the approach of stepwise variable selection, stepping up, only variables with a significance level of 0.05 were included in the model. We used SPSS for Windows 21.0 (SPSS Inc., Chicago, IL) for statistical analyses. In general, $p<0.05$ were considered statistically significant.

\section{RESULTS}

\section{Baseline Characteristics}

The cohort is composed of 172 healthy Finnish children and adolescents, 106 girls and 66 boys, between 7 and 19 years of age. The mean $( \pm \mathrm{SD})$ age was $13.8 \pm 2.7$ years (range $7.4-$ 18.8) for girls and $12.5 \pm 2.7$ years (range 7.7-18.1) for boys (Supplementary Tables 1, 2). As the age distribution in boys differed from that in girls, including more younger subjects, we introduced robust age categories of $8,11,14,17$, and 19 years for girls and corresponding categories for boys, but without the oldest age category (19) (Supplementary Figure 1). For girls (G) and boys (B), the chronological age ranges per age category (minimum-maximum) are as follows: 8 years (G: 7.4-9.0; B: 7.78.9), 11 years (G: 10.5-12.4; B: 10.4-12.4), 14 years (G: 12.515.4; B: 12.5-15.4), 17 years (G: 15.5-18.4; B: 15.4-18.1), and 19 years (G: 18.5-18.8; B: none). The distribution of participants by their (pre-, mid-, and post-) pubertal stages per age category is presented in Figure 1 (girls) and Figure 2 (boys).

Baseline characteristics, including demographics and anthropometric, body composition/DXA and key biochemical parameters confirmed that the cohort represents healthy children and adolescents of normal height (Supplementary Figure 1). The background descriptive data are summarized for each age category in Supplementary Tables 1,2 for girls and boys, respectively.

\section{Other BTMs}

Concentrations for all the introduced BTMs, i.e., formation markers S-P1NP and S-ALP, and resorption markers S-ICTP and U-NTx, increased per age category with puberty or were already elevated prior to it. As expected, the BTM values correlated also with pubertal growth, with declining concentrations per age category observed earlier in girls (after 11 years) than in boys (after 14 years) (Tables 1A,B). The concentrations for these BTMs, for all the age categories, were within age-specific reference intervals and confirmed that the participants had a normal bone turnover at the time of evaluation.

\section{Serum Total and Carboxylated OC}

All the OC concentrations associated with age and puberty (Figures 3A,B,D,E). The mean peak serum total OC values per age category were $38.7 \pm 11.47 \mathrm{ng} / \mathrm{ml}$ (11 years) and $38.8 \pm 9.43 \mathrm{ng} / \mathrm{ml}$ (14 years) in girls and boys, respectively. The peak OC values coincided with the mid-pubertal stage for both sexes (Figures 1, 2), and the dynamics of the opposite trends for total serum OC concentrations were most pronounced in age categories 11 to 14 in girls vs. boys. When entering the post-pubertal stage, the OC values decreased closer to those observed in young adults, and earlier in girls to levels lower vs. those observed in boys at 17 years. The pattern of concentrations of carboxylated OC was consistent with that of total serum OC (Tables $2 A, \mathbf{B}$, Figures 3A-F).

Based on these values, we established pediatric normative reference values for serum total and carboxylated OC per age categories, for girls and boys in Tables 2A,B, respectively. Additionally, Figures 3A,B for girls and Figures 3D,E for boys display the different percentiles (between 10th and 90th) per age category for serum total and carboxylated OC concentrations.

\section{Urinary Mid-Fragment OC}

Contrary to the other BTMs (standard serum and urinary BTMs, and serum OC), the highest concentrations for creatinine corrected urinary mid-fragment OC values were observed in prepubertal children and in the youngest age category (8 years) in both girls and boys (Figures $3 \mathbf{C}, \mathbf{F}$, respectively). In general, there was a strong correlation between the serum total and/or 
TABLE 1A | Serum P1NP, ICTP, ALP, and urinary NTX concentrations per age category for girls.

\begin{tabular}{|c|c|c|c|c|c|c|c|c|c|}
\hline BTM (unit) & Age category (range) & $\boldsymbol{N}$ & Mean & SD & SEM & \multicolumn{2}{|c|}{$95 \% \mathrm{Cl}$} & Min & Max \\
\hline \multirow{3}{*}{ Serum P1NP ( $\mu \mathrm{g} / \mathrm{L})$} & $11(10.5-12.4)$ & 27 & 683.7 & 245.68 & 47.28 & 586.5 & 780.9 & 201.0 & $1,095.0$ \\
\hline & $14(12.5-15.4)$ & 39 & 447.7 & 303.23 & 48.55 & 349.4 & 546.0 & 95.0 & $1,511.0$ \\
\hline & $17(15.5-18.4)$ & 25 & 94.2 & 37.42 & 7.48 & 78.8 & 109.6 & 26.0 & 162.0 \\
\hline \multirow[t]{5}{*}{ Serum ICTP ( $\mu \mathrm{g} / \mathrm{L})$} & $8(7.4-9.0)$ & 7 & 15.3 & 3.82 & 1.44 & 11.8 & 18.8 & 11.0 & 20.0 \\
\hline & $11(10.5-12.4)$ & 27 & 17.3 & 4.76 & 0.92 & 15.4 & 19.1 & 10.0 & 33.0 \\
\hline & $14(12.5-15.4)$ & 39 & 14.9 & 4.58 & 0.73 & 13.4 & 16.4 & 5.0 & 25.0 \\
\hline & $17(15.5-18.4)$ & 25 & 7.1 & 1.96 & 0.39 & 6.3 & 7.9 & 4.0 & 12.0 \\
\hline & 19 (18.5-18.8) & 5 & 5.6 & 0.89 & 0.40 & 4.5 & 6.7 & 5.0 & 7.0 \\
\hline ALP ( $\mu \mathrm{g} / \mathrm{L})$ & $19(18.5-18.8)$ & 5 & 58.8 & 14.08 & 6.30 & 41.3 & 76.3 & 39.0 & 74.0 \\
\hline \multirow[t]{5}{*}{ Urinary NTx (nmol/mol) } & $8(7.4-9.0)$ & 7 & 800.9 & 237.73 & 89.86 & 581.0 & $1,020.7$ & 617.0 & $1,290.4$ \\
\hline & $11(10.5-12.4)$ & 25 & 840.2 & 309.60 & 61.92 & 712.4 & 968.0 & 448.0 & $1,509.2$ \\
\hline & $14(12.5-15.4)$ & 33 & 449.4 & 247.82 & 43.14 & 361.5 & 537.2 & 75.4 & 973.8 \\
\hline & $17(15.5-18.4)$ & 24 & 117.8 & 65.50 & 13.37 & 90.1 & 145.4 & 32.4 & 305.3 \\
\hline & $19(18.5-18.8)$ & 4 & 89.3 & 14.75 & 7.38 & 65.9 & 112.8 & 68.7 & 101.5 \\
\hline
\end{tabular}

TABLE 1B | Serum P1NP, ICTP, ALP, and urinary NTx concentrations per age category for boys.

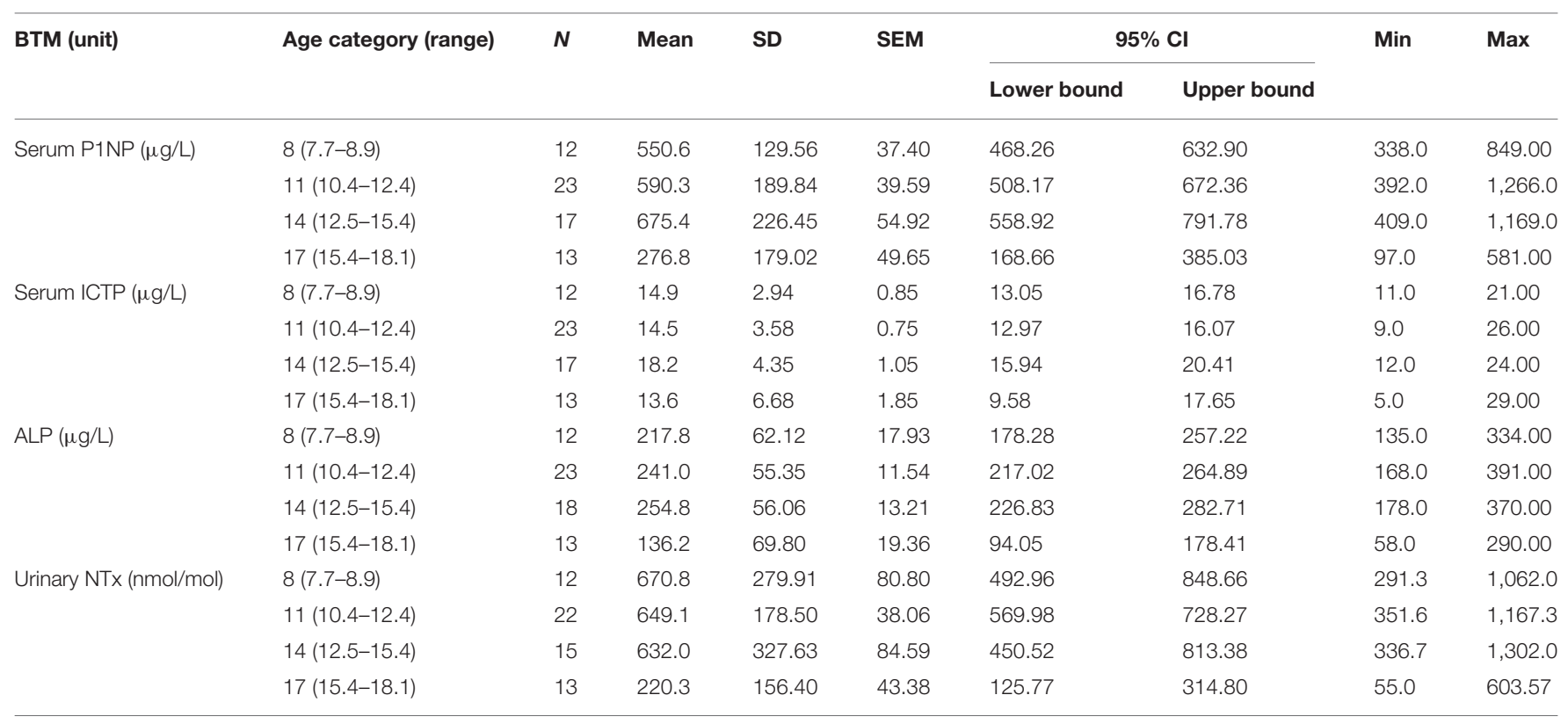

carboxylated OC and urinary mid-fragment OC concentrations in the overall pooled cohort for girls (Spearman correlation $r$ between 0.76 and 0.78 , all $p<0.001$ ). However, for boys, the pooled urinary mid-fragment concentrations lacked correlation between total serum OC and carboxylated OC concentrations ( $r=0.12$ and 0.234 , for total and carboxylated OC, respectively, $p>0.05)$. In boys, the correlation between the total serum and carboxylated and urinary $\mathrm{OC}$ improved progressively with 


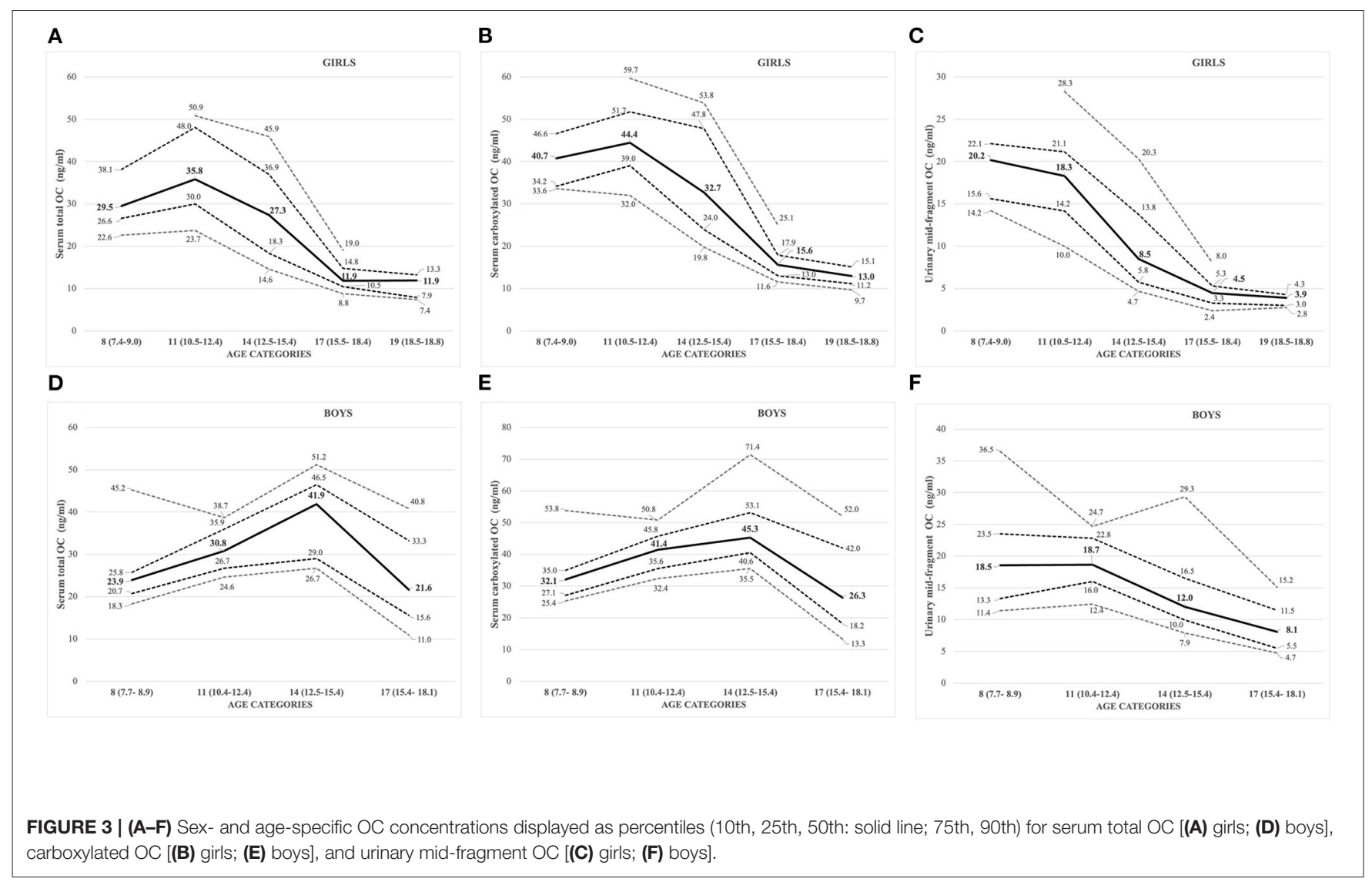

TABLE 2A | Serum total, carboxylated, and urinary mid-fragment OC values as geometrical mean ( $\pm S D$ ) and percentiles for girls.

\begin{tabular}{|c|c|c|c|c|c|c|c|c|}
\hline Osteocalcin ng/ml & Age category (range) & Geometrical mean & SD & Minimum & \multicolumn{3}{|c|}{ Tukey's hinges percentiles } & Maximum \\
\hline & $11(10.5-12.4)$ & 38.7 & 11.47 & 23.4 & 30.0 & 35.8 & 47.1 & 72.3 \\
\hline & $14(12.5-15.4)$ & 28.6 & 12.03 & 11.3 & 18.4 & 27.3 & 36.9 & 54.9 \\
\hline & $17(15.5-18.4)$ & 13.0 & 3.59 & 8.6 & 10.5 & 11.9 & 14.7 & 21.8 \\
\hline \multirow[t]{5}{*}{ Serum carboxylated } & $8(7.4-9.0)$ & 41.5 & 8.05 & 33.6 & 34.7 & 40.0 & 46.5 & 54.9 \\
\hline & $11(10.5-12.4)$ & 45.8 & 9.45 & 29.3 & 39.9 & 44.4 & 51.6 & 67.6 \\
\hline & $14(12.5-15.4)$ & 35.8 & 13.31 & 14.9 & 24.4 & 32.7 & 47.6 & 63.5 \\
\hline & $17(15.5-18.4)$ & 16.6 & 4.80 & 10.4 & 13.1 & 15.6 & 17.7 & 28.8 \\
\hline & $19(18.5-18.8)$ & 13.1 & 2.36 & 9.7 & 12.7 & 13.0 & 14.1 & 16.2 \\
\hline & $19(18.5-18.8)$ & 3.7 & 0.69 & 2.8 & 3.2 & 3.9 & 4.2 & 4.3 \\
\hline
\end{tabular}

increasing age, and the strongest correlations were observed in the oldest age (17) category (correlation with urinary OC: $r=$ 0.692, $p=0.009$ for total OC and $r=0.610, p=0.027$ for carboxylated OC).

\section{Impact of Vitamin D Status and PTH on BTM Concentrations}

Serum samples for assessment of Vitamin D status were available for almost all participants: The mean serum 25-OHD ( $\pm S D$ ) 
TABLE 2B | Serum total, carboxylated, and urinary mid-fragment OC values as geometrical mean ( \pm SD) and percentiles for boys.

\begin{tabular}{|c|c|c|c|c|c|c|c|c|}
\hline \multirow[b]{2}{*}{ Osteocalcin (ng/ml) } & \multirow[b]{2}{*}{ Age category (range) } & \multirow[b]{2}{*}{ Geometrical mean } & \multirow[b]{2}{*}{ SD } & \multirow[b]{2}{*}{ Minimum } & \multicolumn{3}{|c|}{ Tukey's hinges percentiles } & \multirow[b]{2}{*}{ Maximum } \\
\hline & & & & & 25th & 50th & 75th & \\
\hline \multirow[t]{4}{*}{ Serum total } & $8(7.7-8.9)$ & 25.5 & 8.88 & 18.0 & 20.9 & 23.9 & 25.6 & 51.6 \\
\hline & $11(10.4-12.4)$ & 31.5 & 6.18 & 17.8 & 27.3 & 30.8 & 35.6 & 46.4 \\
\hline & $14(12.5-15.4)$ & 38.8 & 9.43 & 26.7 & 29.0 & 39.6 & 46.5 & 53.0 \\
\hline & $17(15.4-18.1)$ & 23.6 & 10.43 & 10.3 & 15.7 & 21.6 & 31.7 & 43.8 \\
\hline \multirow[t]{4}{*}{ Serum carboxylated } & $8(7.7-8.9)$ & 33.6 & 9.39 & 25.4 & 27.2 & 32.1 & 34.9 & 60.1 \\
\hline & $11(10.4-12.4)$ & 40.6 & 6.84 & 26.1 & 35.7 & 41.4 & 44.9 & 52.9 \\
\hline & $14(12.5-15.4)$ & 48.2 & 11.81 & 31.6 & 40.6 & 45.3 & 52.0 & 71.9 \\
\hline & $17(15.4-18.1)$ & 29.4 & 14.03 & 12.2 & 18.8 & 26.3 & 38.3 & 52.8 \\
\hline \multirow[t]{4}{*}{ Urinary mid-fragment } & $8(7.7-8.9)$ & 19.8 & 8.19 & 11.1 & 13.6 & 18.5 & 22.8 & 39.7 \\
\hline & $11(10.4-12.4)$ & 19.1 & 4.89 & 8.8 & 16.3 & 18.7 & 22.6 & 29.8 \\
\hline & $14(12.5-15.4)$ & 15.2 & 7.66 & 6.9 & 10.6 & 12.1 & 16.1 & 32.3 \\
\hline & $17(15.4-18.1)$ & 8.7 & 3.65 & 4.7 & 5.5 & 8.1 & 9.5 & 16.0 \\
\hline
\end{tabular}

concentration was $42.5 \pm 12.5 \mathrm{nmol} / \mathrm{L}$ (range $17.0-82.0 \mathrm{nmol} / \mathrm{L}$ ) for all girls and boys (pooled, $n=171$ ) and below $50 \mathrm{nmol} / \mathrm{L}$ in $70 \%$ (120 out of 170 subjects) of the cohort, indicating high prevalence of Vitamin D insufficiency. An inverse association between 25-OHD and the levels of both serum total and carboxylated OC was only observed in the highest 25-OHD tertile $(r=-0.321, p=0.016$ for total OC and $r=-0.381, p=0.004$, for carboxylated OC). Independent of the 25-OHD tertile, other assessed BTMs were strongly associated with total, carboxylated, and urinary OC $(p<0.001$ for most BTMs, only ICTP not being associated with urinary mid-fragment $\mathrm{OC}$ in the highest 25-OHD tertile).

There was a negative correlation between PTH concentrations and 25-OHD tertiles $(p=0.014)$. The serum total and carboxylated OC concentrations were associated with $\mathrm{PTH}$ in the lowest 25-OHD tertile (Spearman, $r=0.404, p=0.003$, for total OC; and $r=0.384, p=0.005$, for carboxylated OC) but not in the entire cohort.

\section{Individual Determinants of Serum and Urinary OC Concentrations}

We tested factors influencing the serum total, carboxylated, and urinary mid-fragment OC with multivariable linear regression model. Independent determinants for each OC parameter varied; the urinary $\mathrm{OC}$ was the most robust and only influenced by age and puberty, while age, height and weight (marginally for total OC), and PTH (for carboxylated OC) were significant determinants of serum OC values (Supplementary Table 3). Body fat $\%$, BMI, or other evaluated anthropometric, DXA, or biochemical markers had no statistically significant influence on serum or urinary OC values (for all, $p>0.05$ ).

\section{DISCUSSION}

We report, for the first time, a composite set of pediatric reference values for healthy Caucasian children and adolescents for serum total and carboxylated OC and urinary mid-fragment OC. In these young individuals with normal bone health, we demonstrated that age, height, weight, puberty, and PTH are all significant and independent determinants of serum and urinary OC levels during childhood and adolescence. Key markers reflecting overall health or growth status, such as weight and height-adjusted weight, BMI, height SD, or 25-OHD levels, seem to have no or limited significance to OC. Similarly, and rather unexpectedly, the body composition in terms of fat $\%$ or BMD $Z$-score in WB, femoral, or LS showed limited or no association with OC values. While, in adults, BTMs predict BMD values, in children, none of the measured BTMs associated with the BMD Z-score.

\section{External Validation}

Our post-pubertal OC results are comparable or in line with the previously reported mean OC levels in two different cohorts of Finnish young adults $(23,25)$, providing an additional external validation of these data in other reference populations close to their peak bone mass. We also demonstrated a well-established pattern of variation and correlation of the BTM levels with pubertal growth; progressive increase in early puberty and the values for girls declining a few years earlier than for the boys, even if there were differences between some of the evaluated BTMs.

The pattern of dynamics differs for each BTM and may depend on physiological factors that affect these. The turnover marker concentrations also differ regarding which dimension of the bone turnover cycle they reflect. Urinary OC fragments may be derived from new biosynthetic OC or released during degradation of old matrix during bone resorption while circulating $\mathrm{OC}$ is being released from mature osteoblasts during late stages of bone formation (39). As bone modeling is predominantly driving skeletal growth in children and adolescents, the shift to a more pronounced pattern of coupled remodeling in post-pubertal individuals may explain the observed improved correlation between serum and urinary OC in our analysis at time points 
closer to the age of peak bone mass and sexual maturation. In addition, the OC levels in general were strongly driven by age. As age and pubertal stage are closely linked, this progressive increase in correlation between serum and urinary OC values can also be observed when the results are displayed by pubertal stages, reflecting the activation of hormonal maturation in both sexes rather than by age category per se.

\section{Impact of Vitamin D (25-OHD) Status on Markers of Bone Turnover}

Bone mineralization as a process is dependent on adequate dietary intake of calcium and phosphate. In turn, the absorption of calcium is dependent on the intake and levels of active Vitamin D (40). However, controlled studies in adolescents show no relationship between serum 25-OHD and calcium absorption efficiency with higher 25-OHD concentrations (41), but in those with serum 25-OHD concentrations below 50$62.5 \mathrm{nmol} / \mathrm{L}$, as in our cross-sectional cohort, the relationship appears negative (30). In a Vitamin D sufficient state, net calcium absorption is $\sim 30 \%$, and during active growth in pediatric populations, it increases to $60-80 \%$ (42). Vitamin D deficiency leads to diminished levels of calcium and phosphate, the essential constituents for bone mineralization (43). The relationship between Vitamin D and calcium absorption efficiency has not been studied extensively in children and adolescents and only few studies have explored the effect of the nutritional status, especially of 25-OHD on the levels of BTMs, in the otherwise healthy children.

At the time of study, 25-OHD levels were even lower than expected in our healthy pediatric population (mean value, $42.5 \mathrm{nmol} / \mathrm{L}$ ), despite the reported use of supplements in addition to dietary intakes that were in accordance with the national recommendations (30). More recently, the Vitamin $\mathrm{D}$ status in the adult population has significantly improved due to fortification of commonly used foods with Vitamin D and increase in Vitamin D supplementation (44). Nevertheless, investigation of association between 25-OHD status, but also potentially even BMD Z-score, BMC, bone area, and BTMs in a healthy population, is essential for interpretation of the effect of potential sub-optimal bone mineralization on any BTM results.

\section{Adjusting Local Reference BTM Values}

Reliable assessment of pediatric reference data for BTMs requires a representative sample population of healthy children and adolescents within a well-defined geographical area. Many factors show geographical variation and influence BTM reference values, e.g., Vitamin D (in)sufficiency, dietary habits such as milk intake, local recommendations for supplementation of minerals or vitamins, exercise habits, and even religious factors such as veiling practices (45). Hence, every geographical area should ideally have access to locally adjusted reference values. Most of the available BTM data in children and adolescents are only available for post-hoc analysis of birth cohorts, different bone-derived diseases, osteoporosis, or conditions inducing a secondary effect of glucocorticoid therapy on their bone health (46-49). In these populations, assessment of calcium, $\mathrm{PTH}$, and phosphate levels in relation to the BTMs has been demonstrated as crucial for correct interpretation of impaired bone markers and metabolism in children and adolescents. In our cohort, only PTH and 25-OHD besides age, height, and weight were shown to be independent determinants of serum total and carboxylated OC. Our results are therefore aligned with a recent cohort study, which showed that total OC together with other BTMs and hormonal regulation were mainly affected by BMI (50): emphasizing the importance of yet considering the impact of individual body weight on interpretation of biomarkers and exploring long-term consequences of obesity on bone health in increasingly overweight cohorts of children and adolescents in general.

\section{Impact of Sampling Methodology on Results}

When assessing any biological markers in children and adolescence, a preferred sampling method is non-invasive, such as urinary sampling as an alternative to serum sampling. Yet, in our pediatric cohort, the urinary samples were less often retrievable (92 and $94 \%$ for girls and boys, respectively) than serum samples ( $100 \%$ for all). The midfragment urinary OC reflects different aspects of bone turnover, also potentially resorption $(39,51)$, and it has also been demonstrated to be a marker of growth in a special population of prematurely born infants during their postnatal growth spurt (19). However, our study results indicated that there were more uncertainties for use of both serum $\mathrm{OC}$ and urinary OC, especially in younger boys, due to serum OC in youth being mainly derived from de novo biosynthesis and bone formation. The improved correlation with increasing age might therefore simply be an indicator of proportionally increased presence of balanced remodeling and synchronized coupling signals.

\section{Strengths and Limitations of Our Analysis}

The main weakness of our study was also the limited overall number of subjects as a total of 172 children and adolescents are less than recommended by international guidelines for validated assessment of reference intervals (4). In particular, the low number of boys with advanced pubertal stage might partially explain the low correlation between the serum and urinary OC values in growing boys. On the other hand, assessment of both serum and urinary OC values provided valuable information related to the different roles of these two OC components that possibly reflect both bone formation and resorption (52). We also targeted anthropomorphic factors, components of body composition as potential predictors of BTMs and bone health, while we did not include some of the more advanced biomarkers, such as insulin-like growth factor-1 or its binding protein, shown to predict and explain a large proportion of variation in calcium retention in adolescent boys (53). Nevertheless, we have established reference ranges for younger individuals in a relatively large cohort of healthy girls and boys, and a proposal for a range of normal values for those approaching post-pubertal ages, by presenting OC values scattered over different stages of 
puberty and growth spurt, with a confirmatory array of standardized BTMs reflecting bone turnover from childhood toward adulthood and peak bone mass. Our cohort displays characteristics typical for a Caucasian pediatric population located on the northern hemisphere, including low 25-OHD levels in midwinter samples, and therefore providing a platform and reference intervals for clinical interpretation of BTM results in corresponding individuals.

\section{CONCLUSIONS}

The proposed sex- and age-specific reference intervals for serum and urine OC in healthy Finnish children and younger adolescents were mostly influenced by anthropomorphic factors such as age, puberty status, height, and weight. In younger children (age $<11$ ), circulating OC reflects more growth status than bone metabolism per se. Thus, its validity, similar to other BTMs, as a single determinant of healthy pediatric bone status alone might be limited. Yet, BTMs provide a supplementary tool when assessing longitudinal changes in bone health with repeat measurements, in combination with other clinically relevant parameters.

\section{DATA AVAILABILITY STATEMENT}

The original contributions presented in the study are included in the article/Supplementary Material, further inquiries can be directed to the corresponding author/s.

\section{ETHICS STATEMENT}

The Helsinki and Uusimaa Hospital District Independent Ethics Committee approved the study protocol and a written, ageadjusted informed consent was given by the participants and/or their caregivers, as appropriate. The study was carried out according to the principles of Declaration of Helsinki.

\section{REFERENCES}

1. Eastell R, Pigott T, Gossiel F, Naylor KE, Walsh JS, Peel NFA. Diagnosis of endocrine disease: Bone turnover markers: are they clinically useful? Eur J Endocrinol. (2018) 178:R19-31. doi: 10.1530/EJE-17-0585

2. van Coeverden, S. C. C. M., Netelenbos JC, de Ridder CM, Roos JC, Popp-Snijders C, et al. Bone metabolism markers and bone mass in healthy pubertal boys and girls. Clin Endocrinol. (2002) 57:10716. doi: 10.1046/j.1365-2265.2002.01573.x

3. Szulc P, Seeman E, Delmas PD. Biochemical measurements of bone turnover in children and adolescents. Osteoporos Int. (2000) 11:28194. doi: 10.1007/s001980070116

4. Yang L, Grey V. Pediatric reference intervals for bone markers. Clin Biochem. (2006) 39:561-8. doi: 10.1016/j.clinbiochem.2005.11.015

5. Huang Y, Eapen E, Steele S, Grey V. Establishment of reference intervals for bone markers in children and adolescents. Clin Biochem. (2011) 44:7718. doi: 10.1016/j.clinbiochem.2011.04.008

6. Manjón Llorente G, Fernández-Espuelas C, González López JM, Ruiz-Echarri MP, Baldellou Vázquez A. Normal values of bone turnover markers in childhood. An Pediatr. (2004) 60:330-6 doi: 10.1016/S1695-4033(04)78279-X

\section{AUTHOR CONTRIBUTIONS}

OM contributed to the initial acquisition of the clinical data and assessed the participants at baseline. The analytical phase of the OC assays was designed and partially conducted by PP and KI. $\mathrm{PP}$ and $\mathrm{HV}$ analyzed the results. PP drafted the first version of the manuscript. All authors contributed to the conceptual design of the current study, initial interpretation of the data, critical revision of the subsequent versions of the manuscript, have approved the final submitted version, and agreed to be accountable for the content of the work.

\section{FUNDING}

This work was financially supported by the Foundation for Paediatric Research, the Academy of Finland, the Helsinki University Hospital research funds, the Sigrid Juselius Foundation, the Folkhälsan Research Foundation, and Turku University Foundation. The funders had no role in study design, data collection and analysis, decision to publish, or preparation of the manuscript.

\section{ACKNOWLEDGMENTS}

We wish to thank all the children and adolescents and their families who participated in this study. Prof. Sture Andersson is acknowledged for input for the data analyses and Natalia Habilainen-Kirillov for technical assistance for the two-site OC immunoassays. The content of this manuscript has been published in part as part of the academic doctoral thesis of PP (29).

\section{SUPPLEMENTARY MATERIAL}

The Supplementary Material for this article can be found online at: https://www.frontiersin.org/articles/10.3389/fped. 2021.610227/full\#supplementary-material

7. Magarey AM, Boulton TJ, Chatterton BE, Schultz C, Nordin BE, Cockington RA. Bone growth from 11 to 17 years: relationship to growth, gender and changes with pubertal status including timing of menarche. Acta Paediatr. (1999) 88:139-46. doi: 10.1111/j.1651-2227.1999.tb01071.x

8. Gilsanz V, Gibbens DT, Roe TF, Carlson M, Senac MO, Boechat MI, et al. Vertebral bone density in children: effect of puberty. Radiology. (1988) 166:847-50. doi: 10.1148/radiology.166.3.3340782

9. Gilsanz V, Chalfant J, Kalkwarf H, Zemel B, Lappe J, Oberfield S, et al. Age at onset of puberty predicts bone mass in young adulthood. J Pediatr. (2011) 158:52-7. doi: 10.1016/j.jpeds.2010.06.054

10. Crofton PM, Kelnar CJ. Bone and collagen markers in paediatric practice. Int J Clin Pract. (1998) 52:557-65.

11. de Ridder CM, Delemarre-van de Waal HA. Clinical utility of markers of bone turnover in children and adolescents. Curr Opin Pediatr. (1998) 10:4418. doi: 10.1097/00008480-199808000-00020

12. Schonau E, Rauch F. Markers of bone and collagen metabolism-problems and perspectives in paediatrics. Horm Res. (1997) 48:50-9. doi: 10.1159/000191329

13. Bailey DA, McKay HA, Mirwald RL, Crocker PR, Faulkner RA. A six-year longitudinal study of the relationship of physical activity to bone mineral accrual in growing children: the University of 
Saskatchewan bone mineral accrual study. J Bone Miner Res. (1999) 14:1672-9. doi: 10.1359/jbmr.1999.14.10.1672

14. Rizzoli R. Determinants of peak bone mass. Ann Endocrinol. (2006) 67:1145. doi: 10.1016/S0003-4266(06)72564-1

15. Rauchenzauner M, Schmid A, Heinz-Erian P, Kapelari K, Falkensammer G, Griesmacher A, et al. Sex- and age-specific reference curves for serum markers of bone turnover in healthy children from 2 months to 18 years. J Clin Endocrinol Metab. (2007) 92:443-9. doi: 10.1210/jc.2006-1706

16. Kirmani S, Atkinson EJ, Melton LJ III, Riggs BL, Amin S, Khosla S. Relationship of testosterone and osteocalcin levels during growth. $J$ Bone Miner Res. (2011) 26:2212-6. doi: 10.1002/jbmr.421

17. Kanbur NO, Derman O, Sen TA, Kinik E. Osteocalcin. A biochemical marker of bone turnover during puberty. Int J Adolesc Med Health. (2002) 14:23544. doi: 10.1515/IJAMH.2002.14.3.235

18. Ivaska KK, Kakonen SM, Gerdhem P, Obrant KJ, Pettersson K, Vaananen HK. Urinary osteocalcin as a marker of bone metabolism. Clin Chem. (2005) 51:618-28. doi: 10.1373/clinchem.2004.043901

19. Kilpeläinen L, Ivaska KK, Kuiri-Hänninen T, Väänänen HK, Rehfeld JF, Goetze JP, et al. Urinary osteocalcin and serum pro-C-type natriuretic peptide predict linear catch-up growth in infants. J Bone Miner Res. (2012) 7:152835 doi: $10.1002 / j b m r .1596$

20. Goulding A, Jones IE, Taylor RW, Williams SM, Manning PJ. Bone mineral density and body composition in boys with distal forearm fractures: a dual-energy x-ray absorptiometry study. J Pediatr. (2001) 139:50915. doi: $10.1067 / \mathrm{mpd} .2001 .116297$

21. Miller PD. Bone density and markers of bone turnover in predicting fracture risk and how changes in these measures predict fracture risk reduction. Curr Osteoporos Rep. (2005) 3:103-10 doi: 10.1007/s11914-005-0018-6

22. Sabhaney V, Boutis K, Yang G, Barra L, Tripathi R, Tran TT, et al. Bone fractures in children: is there an association with obesity? J Pediatr. (2014) 165:313-8.e1. doi: 10.1016/j.jpeds.2014.04.006

23. Paldánius PM, Ivaska KK, Hovi P, Andersson S, Eriksson JG, Väänänen $\mathrm{K}$, et al. Total and carboxylated osteocalcin associate with insulin levels in young adults born with normal or very low birth weight. PLoS ONE. (2013) 8:e63036. doi: 10.1371/journal.pone.0063036

24. Pirilä S., Taskinen M, Turanlahti M, Kajosaari M, Mäkitie O, SaarinenPihkala UM, et al. Bone health and risk factors of cardiovascular diseasea cross-sectional study in healthy young adults. PLoS ONE. (2014) 9:e108040. doi: 10.1371/journal.pone.0108040

25. Viljakainen H, Ivaska KK, Paldánius P, Lipsanen-Nyman M, Saukkonen T, Pietiläinen $\mathrm{KH}$, et al. Suppressed bone turnover in obesity: a link to energy metabolism? A case-control study. J Clin Endocrinol Metab. (2014) 99:215563. doi: 10.1210/jc.2013-3097

26. Booth SL, Centi AJ, Gundberg C. Bone as an endocrine organ relevant to diabetes. Curr Diab Rep. (2014) 14:556. doi: 10.1007/s11892-014-0556-3

27. Hamann C, Kirschner S, Günther KP, Hofbauer LC. Bone Sweet bone osteoporotic fractures in diabetes mellitus. Nat Rev Endocrinol. (2012) 8:297305. doi: 10.1038/nrendo.2011.233

28. Shanbhogue VV, Mitchell DM, Rosen CJ, Bouxsein ML. Type 2 diabetes and the skeleton: new insights into sweet bones. Lancet Diabetes Endocrinol. (2016) 4:159-173 doi: 10.1016/S2213-8587(15)00283-1

29. Paldánius PM. The Role of Osteocalcin in Human Bone Metabolism and Glucose Homeostasis. [Dissertation], University of Helsinki, Helsinki (Finland) (2017)

30. Pekkinen M, Viljakainen H, Saarnio E, Lamberg-Allardt C, Mäkitie O. Vitamin D is a major determinant of bone mineral density at school age. PLoS ONE. (2012) 7:e40090. doi: 10.1371/journal.pone.0040090

31. Sorva R, Lankinen S, Tolppanen EM, Perheentupa J. Variation of growth in height and weight of children. II. After infancy. Acta Paediatr Scand. (1990) 79:498-6. doi: 10.1111/j.1651-2227.1990.tb11503.x

32. Pere A. Comparison of two methods for transforming height and weight to normality. Ann Hum Biol. (2000) 27:35-45. doi: 10.1080/030144600282361

33. Neinstein LS. Adolescent Health Care: A Practical Guide. Philadelphia, PA: Lippincott Williams \& Wilkins (2008).

34. Käkönen SM Hellman J, Karp M, Laaksonen P, Obrant KJ, Väänänen HK, Lövgren T, et al. Development and evaluation of three immunofluorometric assays that measure different forms of osteocalcin in serum. Clin Chem. (2000) 46:332-7. doi: 10.1093/clinchem/46.3.332
35. Hellman J, Kakonen SM, Matikainen MT, Karp M, Lovgren T, Väänänen HK, et al. Epitope mapping of nine monoclonal antibodies against osteocalcin: combinations into two-site assays affect both assay specificity and sample stability. J Bone Miner Res. (1996) 11:1165-75. doi: 10.1002/jbmr.56501 10816

36. Viljakainen HT, Palssa A, Kärkkäinen M, Jakobsen J, Cashman KD, Mølgaard $\mathrm{C}$, et al. A seasonal variation of calcitropic hormones, bone turnover and bone mineral density in early and mid-puberty girls - a cross-sectional study. $\mathrm{Br} \mathrm{J}$ Nutr. (2006) 96:124-30. doi: 10.1079/BJN20061719

37. Zemel BS, Kalkwarf HJ, Gilsanz V, Lappe JM, Oberfield S, Shepherd JA, et al. Revised reference curves for bone mineral content and areal bone mineral density according to age and sex for black and non-black children: results of the bone mineral density in childhood study. J Clin Endocrinol Metab. (2011) 96:3160-9. doi: 10.1210/jc.2011-1111

38. Leonard CM, Roza MA, Barr RD, Webber CE. Reproducibility of DXA measurements of bone mineral density and body composition in children. Pediatr Radiol. (2009) 39:148-54. doi: 10.1007/s00247-008-1067-7

39. Ivaska KK, Hentunen TA, Vääräniemi J, Ylipahkala H, Pettersson K, Väänänen HK. Release of intact and fragmented osteocalcin molecules from bone matrix during bone resorption in vitro. J Biol Chem. (2004) 279:183619. doi: 10.1074/jbc.M314324200

40. Abrams SA, Hicks PD, Hawthorne KM. Higher serum 25-hydroxyvitamin $\mathrm{D}$ levels in school-age children are inconsistently associated with increased calcium absorption. J Clin Endocrinol Metab. (2009) 94:2421-7. doi: 10.1210/jc.2008-2587

41. Lewis RD, Laing EM, Hill Gallant KM, Hall DB, McCabe GP, Hausman $\mathrm{DB}$, et al. A randomized trial of vitamin D3 supplementation in children: dose-response effects on vitamin D metabolites and calcium absorption. J Clin Endocrinol Metab. (2013) 98:4816-25. doi: 10.1210/jc.201 3-2728

42. Holick M. Sunlight and vitamin D for bone health and prevention of autoimmune diseases, cancers, cardiovascular disease. Am J Clin Nutr. (2004) 80:S1678-88. doi: 10.1093/ajcn/80.6.1678S

43. Dietzen DJ, Bennett MJ, Wong ECC (eds). Biochemical and Molecular Basis of Pediatric Disease, 4th Edn. Washington, DC: AACC Press (2010).

44. Raulio S, Erlund I, Männist,ö S., Sarlio-Lähteenkorva S, Sundvall J, Tapanainen $\mathrm{H}$, et al. Successful nutrition policy: improvement of vitamin D intake and status in finnish adults over the last decade. Eur J Public Health. (2017) 27:268-73. doi: 10.1093/eurpub/ckw154

45. Hannon R, and Eastell R. Preanalytical variability of biochemical markers of bone turnover. Osteoporos Int. (2000) 11:S30-44. doi: 10.1007/s0019800 70004

46. Thiering E, Brüske I, Kratzsch J, Hofbauer LC, Berdel D, von Berg A, et al. Associations between serum 25-hydroxyvitamin D and bone turnover markers in a population based sample of German children. Sci Rep. (2015) 15:18138. doi: $10.1038 /$ srep 18138

47. Doyon A, Fischer DC, Bayazit AK, Canpolat N, Duzova A, Sözeri B, et al. Markers of bone metabolism are affected by renal function and growth hormone therapy in children with chronic kidney disease. PLoS ONE. (2015) 10:e01134820. doi: 10.1371/journal.pone.0113482

48. Poyrazoglu S, Gunoz H, Darendeliler F, Bas F, Tutunculer F, Eryilmaz SK, et al. Successful results of pamidronate treatment in children with osteogenesis imperfecta with emphasis on the interpretation of bone mineral density for local standards. J Pediatr Orthop. (2008) 28:4837. doi: 10.1097/BPO.0b013e318173a923

49. Tuchman S, Thayu M, Shults J, Zemel BS, Burnham JM, Leonard MB. Interpretation of biomarkers of bone metabolism in children: impact of growth velocity and body size in healthy children and chronic disease. J Pediatr. (2008) 153:484-90. doi: 10.1016/j.jpeds.2008. 04.028

50. Geserick M, Vogel M, Eckelt F, Schlingmann M, Hiemisch A, Baber $\mathrm{R}$, et al. Children and adolescents with obesity have reduced serum bone turnover markers and 25-hydroxyvitamin $\mathrm{D}$ but increased parathyroid hormone concentrations - results derived from new pediatric reference ranges. Bone. (2020) 132:115124. doi: 10.1016/j.bone.2019. 115124

51. Åkesson K, Käkönen SM, Josefsson PO, Karlsson MK, Obrant KJ, Pettersson $\mathrm{K}$. Fracture-induced changes in bone turnover: a potential confounder in 
the use of biochemical markers in osteoporosis. J Bone Miner Metab. (2005) 23:30-35. doi: 10.1007/s00774-004-0537-8

52. Eastell R, Mallinak N, Weiss S, Ettinger M, Pettinger M, Cain D, et al. Biological variability of serum and urinary N-telopeptides of type I collagen in postmenopausal women. J Bone Miner Res. (2000) 15:5948. doi: 10.1359/jbmr.2000.15.3.594

53. Hill KM, Braun M, Kern M, Martin BR, Navalta JW, Sedlock DA, et al. Predictors of calcium retention in adolescent boys. J Clin Endocrinol Metab. (2008) 93:4743-8. doi: 10.1210/jc.2008-0957

Conflict of Interest: The authors declare that the research was conducted in the absence of any commercial or financial relationships that could be construed as a potential conflict of interest.
Publisher's Note: All claims expressed in this article are solely those of the authors and do not necessarily represent those of their affiliated organizations, or those of the publisher, the editors and the reviewers. Any product that may be evaluated in this article, or claim that may be made by its manufacturer, is not guaranteed or endorsed by the publisher.

Copyright $\odot 2021$ Paldánius, Ivaska, Mäkitie and Viljakainen. This is an open-access article distributed under the terms of the Creative Commons Attribution License (CC $B Y)$. The use, distribution or reproduction in other forums is permitted, provided the original author(s) and the copyright owner(s) are credited and that the original publication in this journal is cited, in accordance with accepted academic practice. No use, distribution or reproduction is permitted which does not comply with these terms. 